\title{
Exophytic Tumor on the Buttock
}

Jane S. Zhang, MD; Joshua B. Kentosh, DO

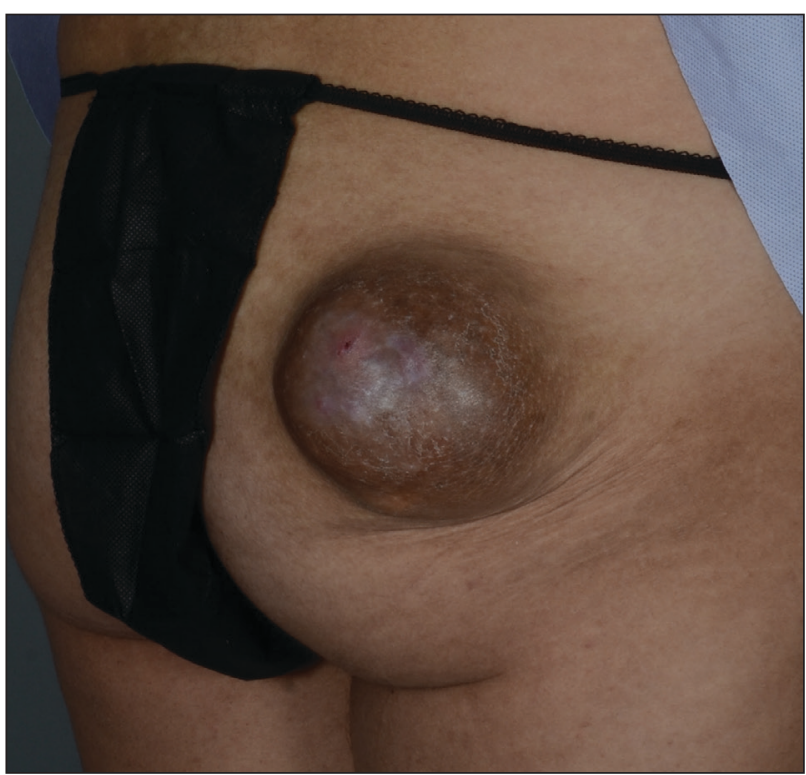

A 20-year-old woman with no notable medical history presented to the dermatology clinic with an enlarging mass on the right buttock that had been growing over the course of several years. The mass progressed from a small, mildly tender nodule to a $10 \times 10-\mathrm{cm}$, hyperpigmented, exophytic tumor. There were no other abnormal findings on physical examination, and the patient denied any systemic symptoms.

\section{WHAT'S YOUR DIAGNOSIS?}
a. cutaneous Crohn disease
b. hidradenocarcinoma
c. lymphangioma
d. Merkel cell carcinoma
e. spiradenoma

From the University of Illinois College of Medicine at Peoria. Dr. Kentosh is from the Department of Dermatology. Dr. Kentosh also is from the Soderstrom Skin Institute, Peoria.

The authors report no conflict of interest.

Correspondence: Jane S. Zhang, MD (jzhan28@uic.edu).

doi:10.12788/cutis.0310 


\section{THE DIAGNOSIS:}

\section{Hidradenocarcinoma}

A n excisional biopsy revealed a neoplasm in the dermis with focal invasion into the adjacent soft tissue (Figure 1). The tumor consisted of sheets of cells with cytoplasmic vacuoles and ductal differentiation (Figure 2), as well as cells with mild atypia, mild pleomorphism, rare mitotic figures, and abundant pale cytoplasm. Immunohistochemical staining was positive for cytokeratin (CK) 5, CK7, CK20, CK AE1/AE3, and p63 (Figure 3). The culmination of features including the large tumor size, immunohistochemical staining pattern, and mild pleomorphism with focal invasion into the soft tissue supported the diagnosis of hidradenocarcinoma.

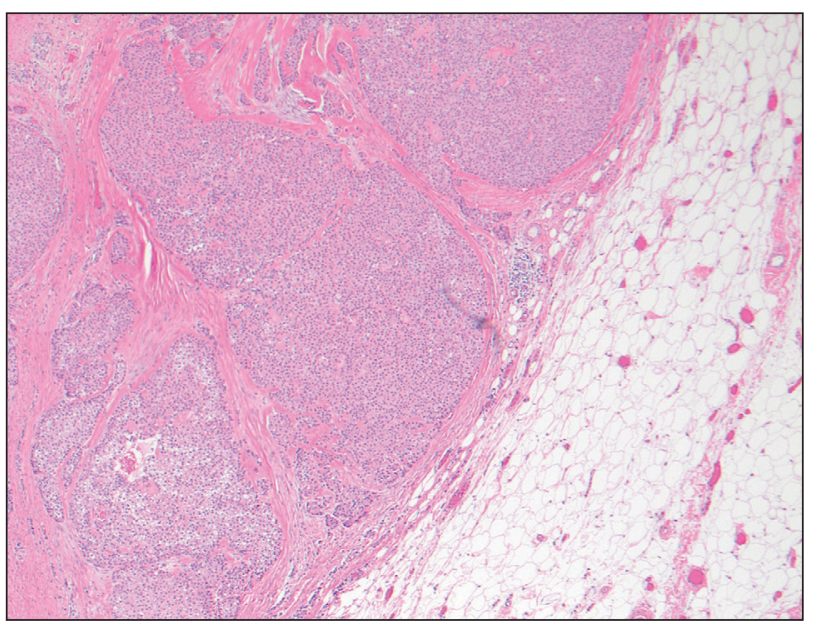

FIGURE 1. Focal invasion into the adjacent soft tissue (H\&E, original magnification $\times 40$ ).

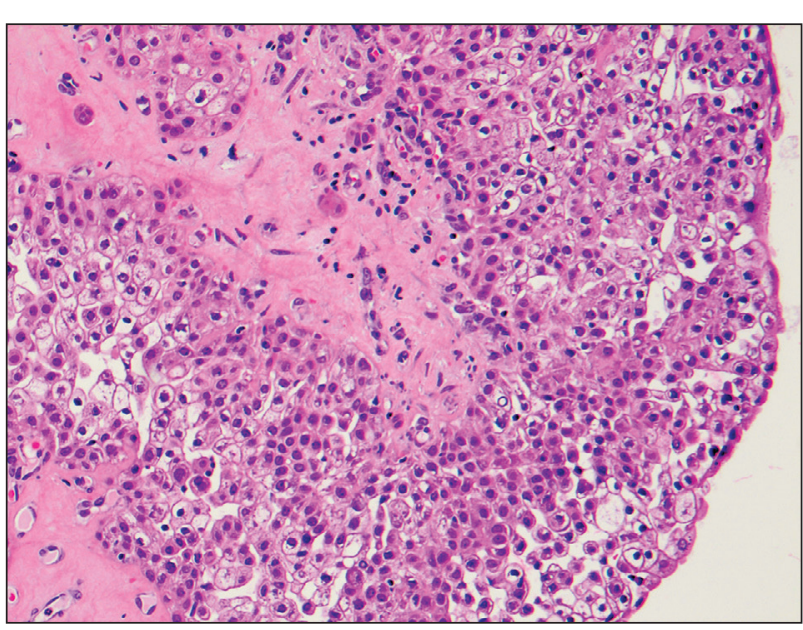

FIGURE 2. Sheets of vacuolated cells with ductal differentiation, mild cellular atypia, and a few sporadic mitotic figures (H\&E, original magnification $\times 200$ ).
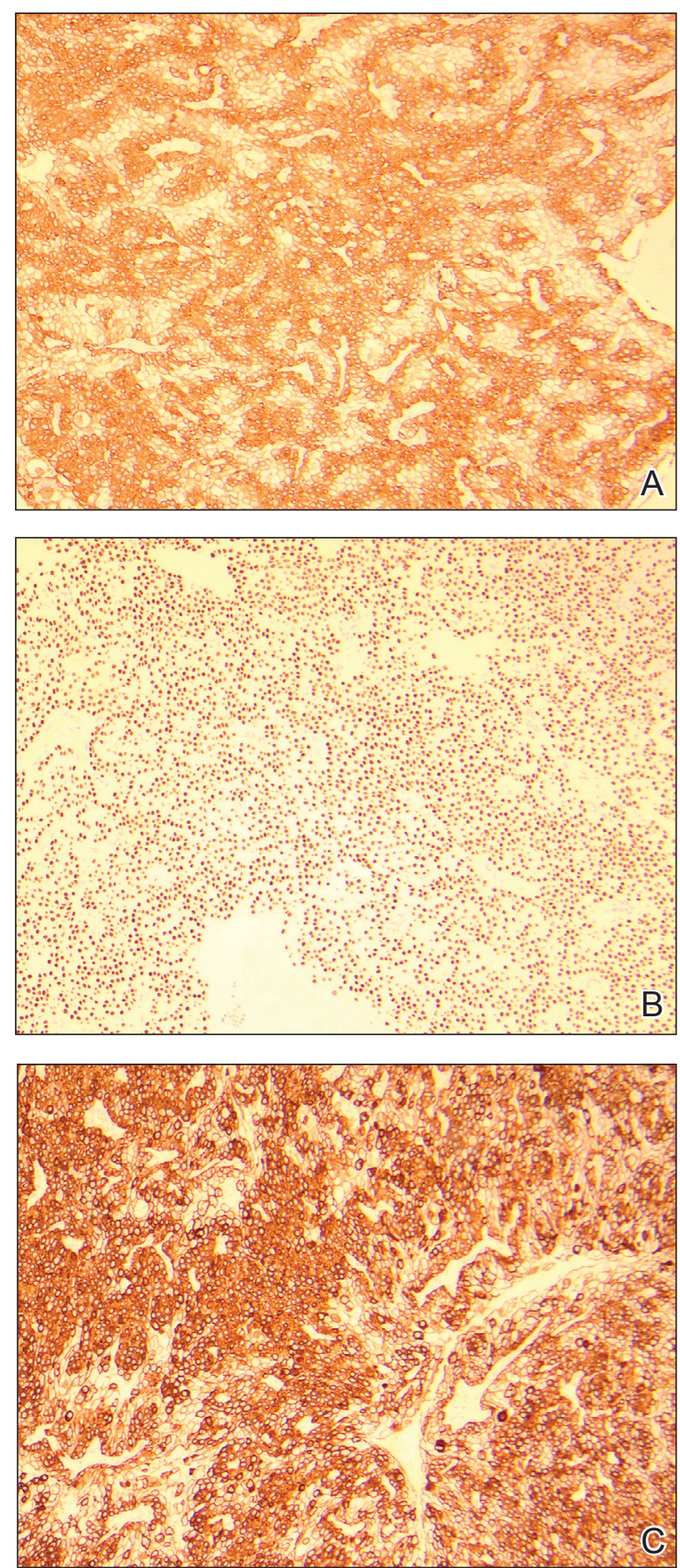

FIGURE 3. A-C, Immunohistochemical staining was positive for cytokeratin AE1/AE3, p63, and cytokeratin 7, respectively (all original magnifications $\times 100)$. 
Hidradenocarcinoma is an exceedingly rare malignant tumor of eccrine and/or apocrine origin. ${ }^{1}$ It accounts for less than $0.001 \%$ of all tumors and 1 of 13,000 skin biopsies. ${ }^{2}$ It usually arises in the head and neck region and most commonly affects older adults aged 50 to 70 years. ${ }^{3}$ The size of hidradenocarcinomas can vary; however, they typically are large, often growing to be greater than $5 \mathrm{~cm}$ in diameter. ${ }^{2}$ It tends to be an aggressive tumor that generally spreads to regional lymph nodes and distant viscera. ${ }^{4}$ Although it most commonly arises de novo, it may occasionally derive from a benign hidradenoma. ${ }^{1}$ The diagnosis of hidradenocarcinoma is made based on the tumor's morphologic and pathologic characteristics. Histologically, it is characterized by an infiltrative and invasive proliferation of lobules made of large clear cells with atypical mitotic figures and nuclear pleomorphism as well as immunohistochemical features displaying various positive markers, such as carcinoembryonic antigen, epithelial membrane antigen, S-100 protein, and CKs AE1/AE3 and 5/6. Invasion of the adjacent soft tissue can be present and helps to confirm the diagnosis.

The differential diagnosis for hidradenocarcinoma primarily is the benign hidradenoma, which is similar both clinically and histologically with a few important differences. Hidradenocarcinomas often are larger and ulcerated. Histologically, they usually are more pleomorphic with the presence of mitotic figures in clear cells and tend to invade locally into the surrounding soft tissue. Other similar lesions such as spiradenoma, Merkel cell carcinoma, lymphangioma, cutaneous Crohn disease, tumors metastatic to the skin, and metastatic clear cell carcinomas originating from other organs also are included in the differential diagnosis. ${ }^{2}$

Spiradenomas are dermal tumors originating from the sweat glands. They typically present as bluish, painful, solitary nodules on the ventral surfaces of the upper body, though multiple nodules also are reported. ${ }^{5}$ Spiradenomas manifest as a central constellation of pale large cells surrounded by small, dark, basaloid cells containing hyperchromatic nuclei. The microscopic appearance of the blue basaloid cells contrasts with the clear cells seen in hidradenoma. ${ }^{5}$

Merkel cell carcinoma is a cutaneous neuroendocrine tumor affecting elderly or immunosuppressed individuals. It arises in sun-exposed areas and often is associated with Merkel cell polyomavirus infection. The histologic features display small and round cells that stain positive for CK8, CK18, CK19, and CK20 but stain negative for CK7, a marker that often is positive in hidradenocarcinoma. ${ }^{6}$

Lymphangioma, particularly cavernous lymphangioma, may resemble the gross appearance of hidradenoma/ hidradenocarcinoma. It usually presents as irregular clear blue papules and nodules in the skin and subcutaneous tissue. ${ }^{7}$ The key histopathologic finding in this tumor is the endothelium-lined channels that stain positive for D2-40, a lymphatic endothelium marker., ${ }^{7,8}$

Cutaneous Crohn disease is classified as noncaseating granulomatous skin lesions that are noncontinuous with the gastrointestinal tract. ${ }^{9}$ Clinical presentations in addition to skin edema include erythematous plaques, ulcerations, and erosions. Histopathology reveals sterile noncaseating granulomas made of Langerhans giant cells, epithelioid histocytes, and plasma cells. ${ }^{9}$

Metastatic clear cell carcinomas, such as renal cell carcinoma, can be differentiated by a history of primary carcinoma, demonstration of histologic vascular stroma, and other features related to metastatic clear cell carcinoma. ${ }^{2}$

There are no well-established therapeutic guidelines for hidradenocarcinoma. Wide local excision with margins greater than $2 \mathrm{~cm}$ is the preferred initial treatment and often is performed in conjunction with sentinel lymph node biopsy. External beam radiotherapy and adjunctive chemotherapy have been used for tumors that could not be surgically cleared. However, the efficacy of these treatments has not been well established. ${ }^{2}$ Targeted therapies recently have emerged as an alternative treatment choice for hidradenocarcinoma due to the utilization of immunohistochemical and genomic testing. The discovery of specific gene mutations or the expression of hormonal receptors in this tumor have paved the way for targeting HER2-expressing hidradenocarcinomas with trastuzumab and those expressing estrogen receptor with the estrogen receptor inhibitor tamoxifen. ${ }^{1}$ Epidermal growth factor receptor inhibitors and PI3K/Akt/mTOR (phosphatidylinositol-3-kinase/AKT/mammalian target of rapamycin) pathway inhibitors also have been used to target various signal transduction pathways. ${ }^{2}$

Wide excision with $2.5-\mathrm{cm}$ margins was performed on our patient, and a positron emission tomographycomputed tomography scan revealed no metastatic disease. She declined sentinel lymph node biopsy and additional treatment. Due to the risk for recurrence, she was monitored closely with skin examinations and positron emission tomography-computed tomography every 3 months for the first year and every 6 months thereafter. Thus far, she has had no evidence of local or regional recurrence.

\section{REFERENCES}

1. Miller DH, Peterson JL, Buskirk SJ, et al. Management of metastatic apocrine hidradenocarcinoma with chemotherapy and radiation. Rare Tumors. 2015;7:6082.

2. Soni A, Bansal N, Kaushal V, et al. Current management approach to hidradenocarcinoma: a comprehensive review of the literature. Ecancermedicalscience. 2015;9:517.

3. Jinnah AH, Emory CL, Mai NH, et al. Hidradenocarcinoma presenting as soft tissue mass: case report with cytomorphologic description, histologic correlation, and differential diagnosis. Diagn Cytopathol. 2016;44:438-441.

4. Khan BM, Mansha MA, Ali N, et al. Hidradenocarcinoma: five years of local and systemic control of a rare sweat gland neoplasm with nodal metastasis. Cureus. 2018;10:E2884.

5. Miceli A, Ferrer-Bruker SJ. Spiradenoma. StatPearls. StatPearls Publishing; 2019

6. Banks PD, Sandhu S, Gyorki DE, et al. Recent insights and advances in the management of Merkel cell carcinoma. J Oncol Pract. 2016; 12:637-646

7. Flanagan BP, Helwig EB. Cutaneous lymphangioma. Arch Dermatol. 1977;113:24-30.

8. Kalof AN, Cooper K. D2-40 immunohistochemistry-so far! Adv Anat Pathol. 2009;16:62-64.

9. Schneider SL, Foster K, Patel D, et al. Cutaneous manifestations of metastatic Crohn's disease. Pediatr Dermatol. 2018;35:566-574. 